Supplementary Information

\title{
Dry Exfoliation of Large-Area 2D Monolayer and
}

\section{Heterostructure Arrays}

Zhiwei Lił, Liwang Rent, Shiyu Wang, Xinxin Huang, Qianyuan Li, Zheyi Lu, Shuimei Ding,

Hanjun Deng, Pingan Chen, Jun Lin, Yuanyuan Hu, Lei Liao, Yuan Liu*

Key Laboratory for Micro-Nano Optoelectronic Devices of Ministry of Education, School of

Physics and Electronics, Hunan University, Changsha 410082, China.

Contents

1. Photos of large-area monolayer $\mathrm{MoS}_{2}$ (Fig. S1).

2. Reusing Au mesh tape for exfoliation (Fig. S2).

3. Schematics and Raman mapping of exfoliated arrays with different square size (Fig. S3).

4. Raman mapping of exfoliated arrays (Fig. S4).

5. Electrical characterizations of monolayer $\mathrm{MoS}_{2}$ arrays (Fig. S5).

6. PL and Raman spectra of monolayer $\mathrm{WSe}_{2}$ arrays, $\mathrm{MoSe}_{2}$ arrays, $\mathrm{WS}_{2}$ arrays (Fig. S6).

7. $\mathrm{WSe}_{2} / \mathrm{MoS}_{2}$ heterojunction preparation process and AFM measurement result (Fig. S7). 
8. Optical image and spectra of multilayer vdWH arrays (Fig. S8, S9).

9. Thickness measurement of PVA functionalization layer (Fig. S10).

10. Dry exfoliation yield of substrate with or without functionalization layer (Fig. S11).
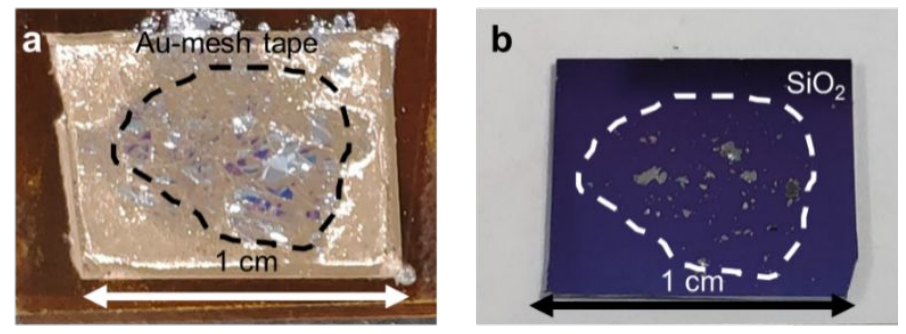

Supplementary Figure 1. Photos of monolayer $\mathrm{MoS}_{2}$ region (by dash line, with random bulk crystals) on Au-mesh tape (a) and released monolayer $\mathrm{MoS}_{2}$ arrays on $\mathrm{SiO}_{2}$ substrate (b).
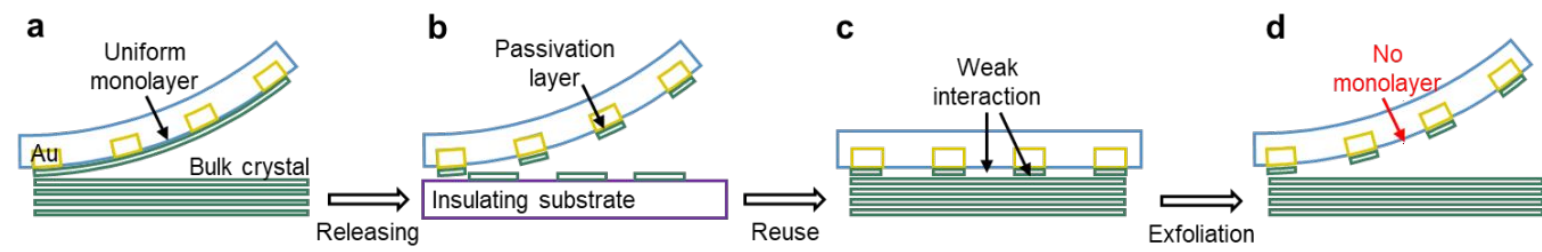

Supplementary Figure 2. Schematical illustration of reusing Au mesh tape for exfoliation. a, Schematic of the mechanical exfoliation of a continuous and uniform monolayer $\mathrm{MoS}_{2}$ using Aumesh tape. b. Schematic of the releasing process, where the non-contact region (between the mesh holes and $\mathrm{MoS}_{2}$ ) ensures weak interaction to mechanically release the $\mathrm{MoS}_{2}$ monolayers on PVA functionalized $\mathrm{SiO}_{2}$ substrate, and the monolayer $\mathrm{MoS}_{2}$ in Au-contact region would remain on the Au surface as a passivation layer. c, d, Schematics of reusing the Au-mesh tape for further exfoliations, where no Au region can contact with the bulk $\mathrm{MoS}_{2}$ crystals, resulting in low exfoliation yield. 

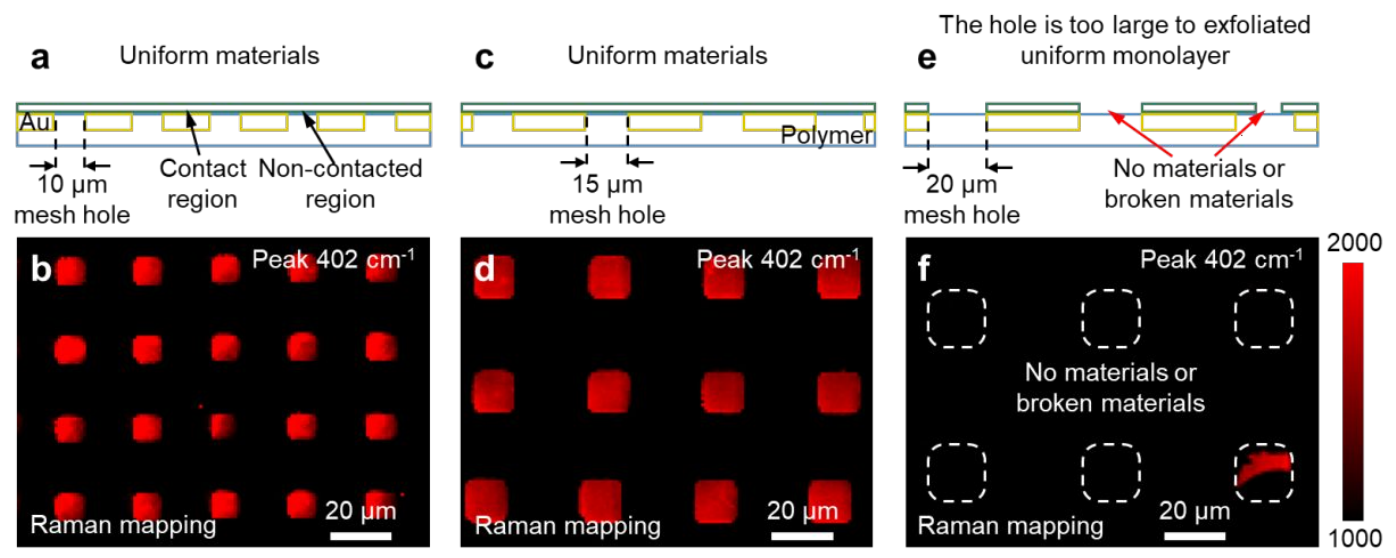

Supplementary Figure 3. a, b, Uniform $\mathrm{MoS}_{2}$ exfoliation on Au-mesh tape with $10 \times 10 \mu \mathrm{m}^{2}$ hole sizes. c, d, Uniform $\mathrm{MoS}_{2}$ exfoliation on Au-mesh tape with hole sizes increase to $15 \times 15 \mu \mathrm{m}^{2}$. e, f, When further increasing the hole size to $20 \times 20 \mu \mathrm{m}^{2}$, the non-contacted region (between polymer and bulk crystal) is too large to exfoliate continuous monolayer, leading to the break of $\mathrm{MoS}_{2}$ flake with low exfoliation yield. We note that the Raman intensity of the monolayer $\mathrm{MoS}_{2}$ in the Au contact region is severely quenched.
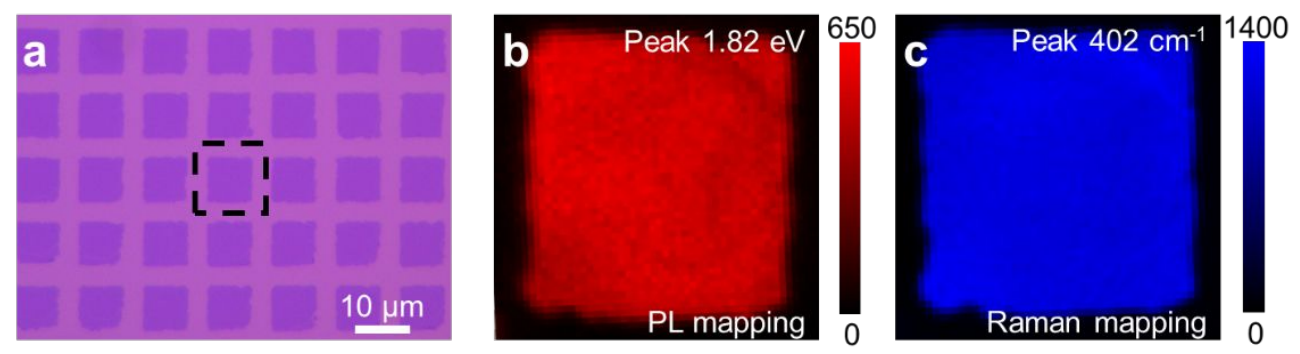

Supplementary Figure 4. a, Optical image of monolayer $\mathrm{MoS}_{2}$ arrays. b, c, PL and Raman intensity mappings with higher resolution (0.2 $\mu \mathrm{m}$ step length) of the monolayer $\mathrm{MoS}_{2}$, demonstrating high uniformity. 

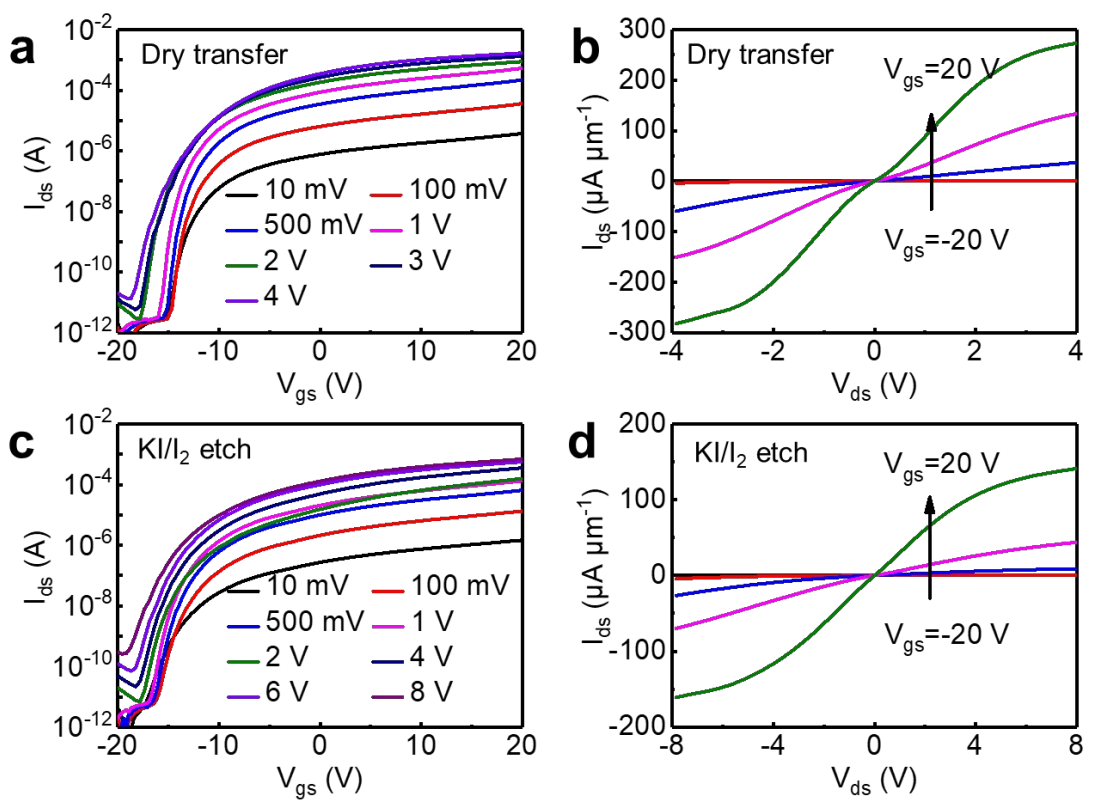

Supplementary Figure 5. Electrical characterizations of monolayer $\mathrm{MoS}_{2}$ arrays. a, b, Transfer and output curves of dry transfer monolayer $\mathrm{MoS}_{2}$ device, showing highest on-state current density of $280 \mu \mathrm{A} \mu \mathrm{m}^{-1}$ while retaining on/off ratio over $10^{8}$. c, d, Transfer and output curves of $\mathrm{KI} / \mathrm{I}_{2}$ etched monolayer $\mathrm{MoS}_{2}$ device, showing highest on-state current density of $160 \mu \mathrm{A} \mu \mathrm{m}^{-1}$.
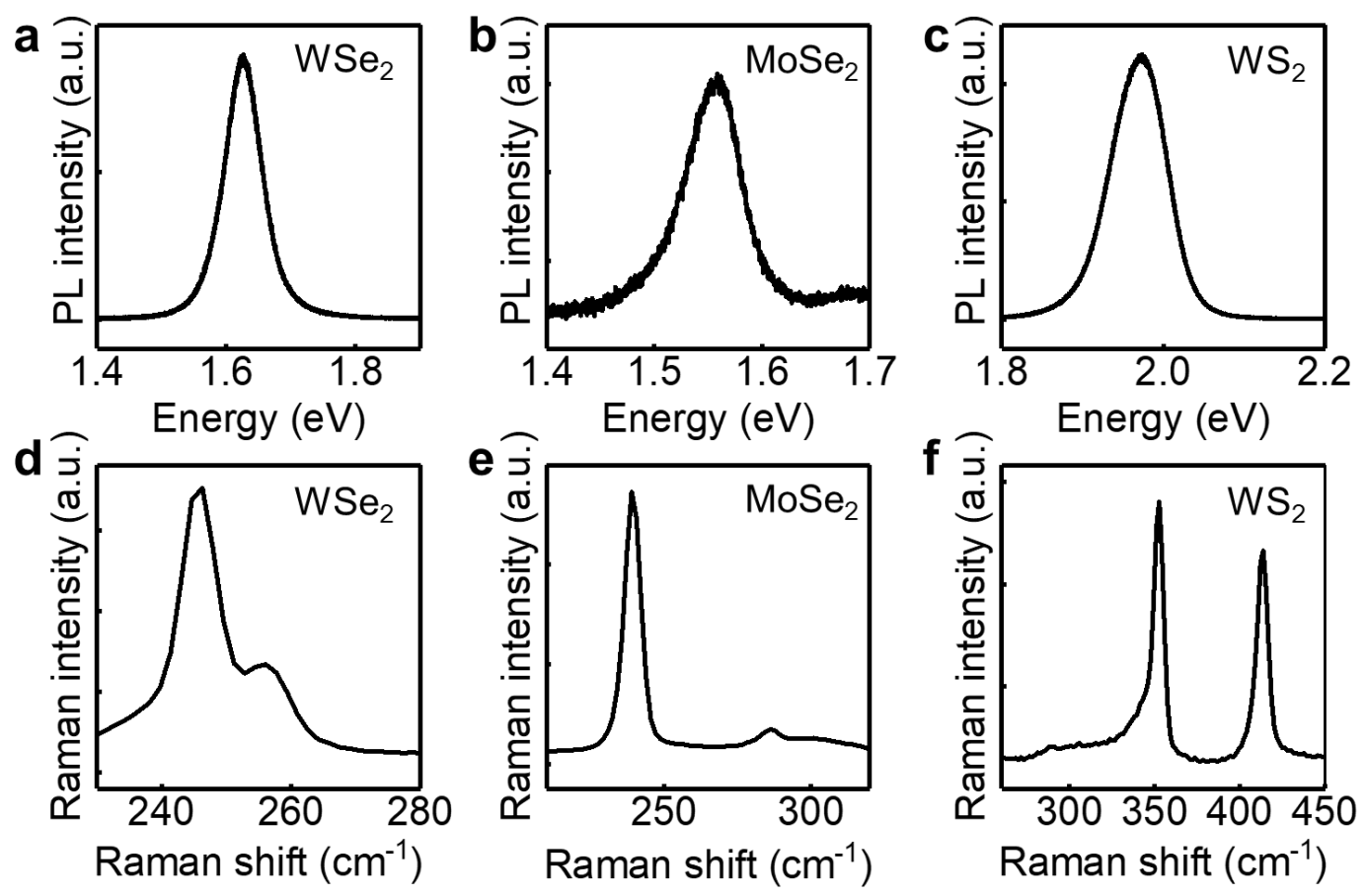
Supplementary Figure 6. PL and Raman spectra of monolayer $\mathrm{WSe}_{2}$ arrays, $\mathrm{MoSe}_{2}$ arrays, $\mathrm{WS}_{2}$ arrays. a-c, PL spectra of monolayer $\mathrm{WSe}_{2}$ arrays, $\mathrm{MoSe}_{2}$ arrays, $\mathrm{WS}_{2}$ arrays. d-f, Raman spectra of monolayer $\mathrm{WSe}_{2}$ arrays, $\mathrm{MoSe}_{2}$ arrays, $\mathrm{WS}_{2}$ arrays.

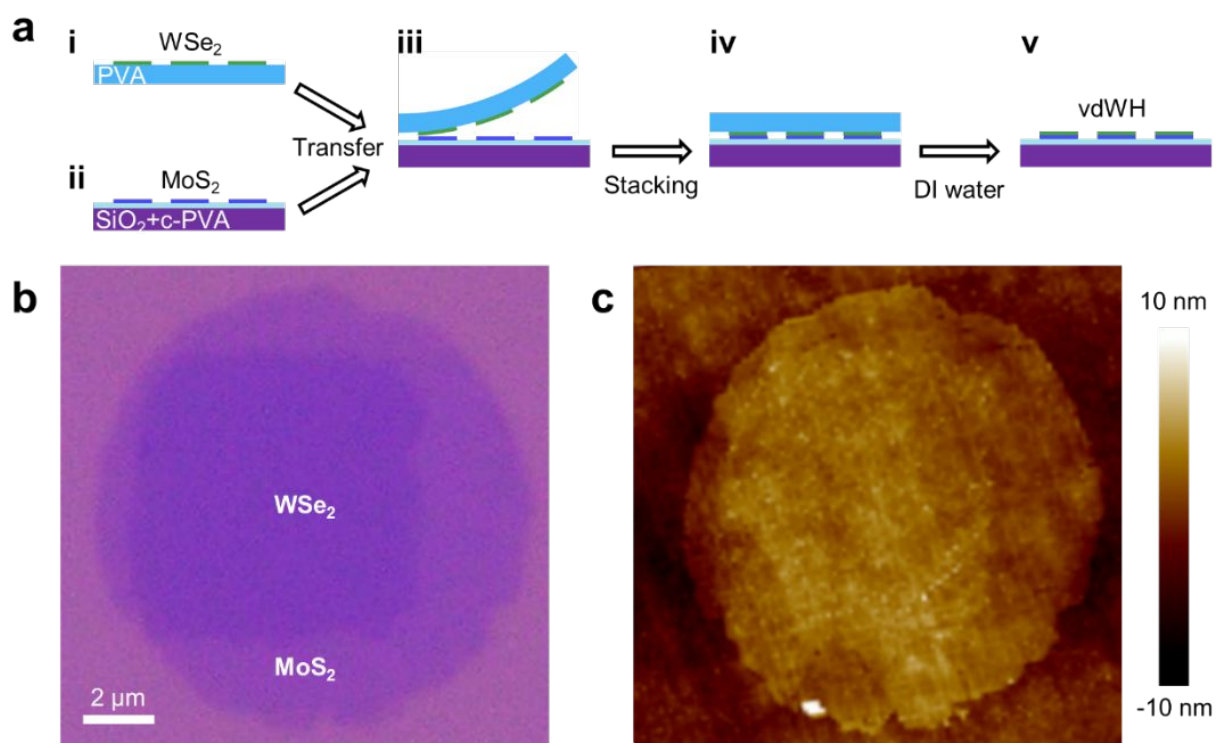

Supplementary Figure 7. a, Schematical illustration of $\mathrm{WSe}_{2} / \mathrm{MoS}_{2}$ heterojunction preparation process with five steps. i), Exfoliation of large-area monolayer $\mathrm{WSe}_{2}$ arrays on PVA substrate. ii), Exfoliation of large-area monolayer $\mathrm{MoS}_{2}$ arrays on c-PVA/SiO${ }_{2}$ substrate. iii, iv) The $\mathrm{WSe}_{2}$ arrays are transferred and vdW integrated on top the $\mathrm{MoS}_{2}$ arrays. v) Removing the top PVA supporting layer in water. b, c, Optical image of a $\mathrm{WSe}_{2} / \mathrm{MoS}_{2} \mathrm{vdWH}$ and its corresponding AFM measurement result, demonstrating the high-quality interface.

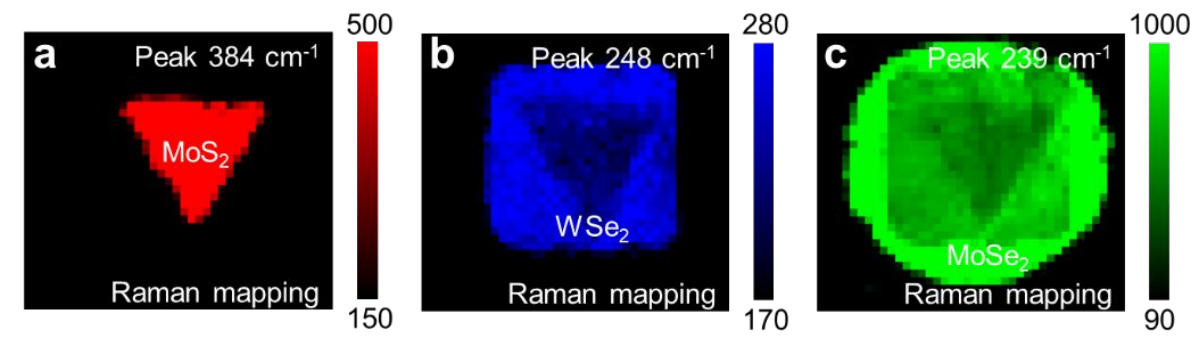


Supplementary Figure 8. Individual Raman intensity mappings of 3-layer $\mathrm{MoSe}_{2} / \mathrm{WSe}_{2} / \mathrm{MoS}_{2}$ vdWH. a, Raman mapping of $1^{\text {st }}$ layer $\mathrm{MoS}_{2} \cdot \mathbf{b}$, Raman mapping of $2^{\text {nd }}$ layer $\mathrm{WSe}_{2}$. c, Raman mapping of $3^{\text {rd }}$ layer $\mathrm{MoSe}_{2}$.
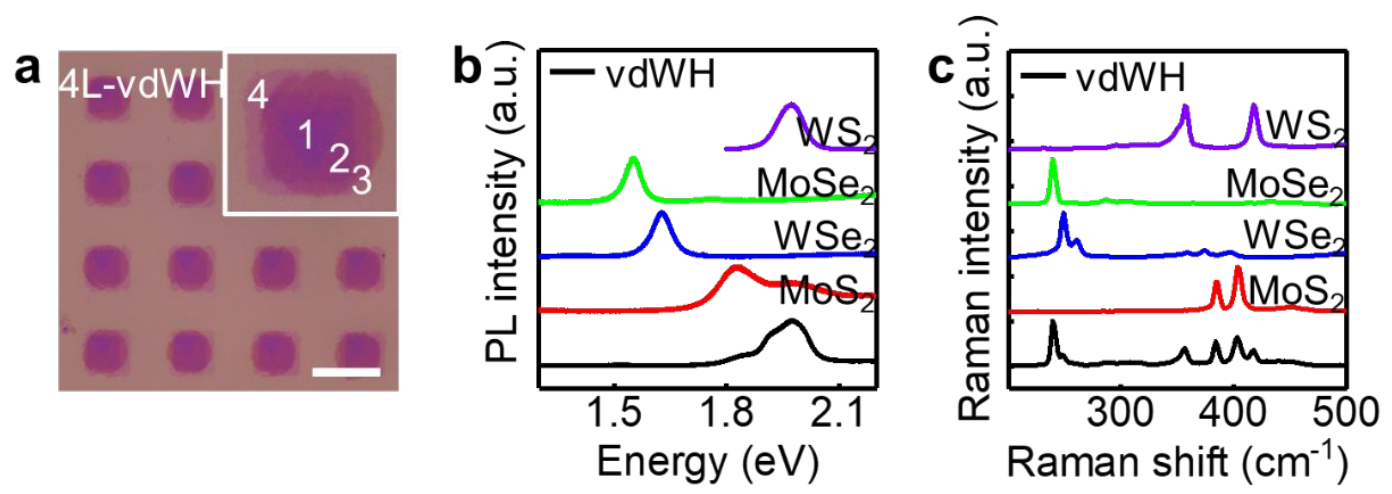

Supplementary Figure 9. Optical image and spectra of 4 -layer $\mathrm{WS}_{2} / \mathrm{MoSe}_{2} / \mathrm{WSe}_{2} / \mathrm{MoS}_{2} \mathrm{vdWH}$ arrays. a, Optical image of 4-layer vdWH arrays, Scale bar: $20 \mu \mathrm{m}$. b, PL spectra of 4-layer vdWH arrays. c, Raman spectra of 4-layer vdWH arrays.

a

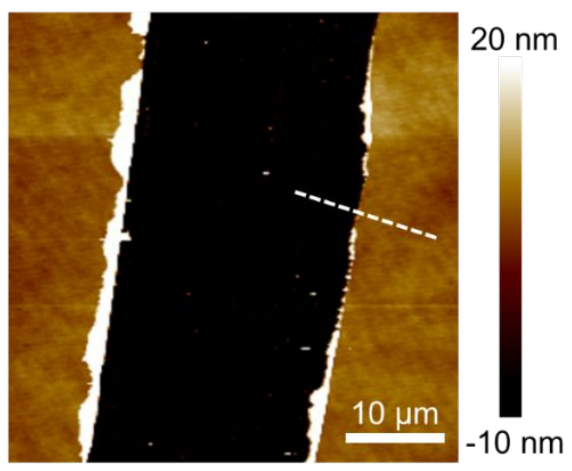

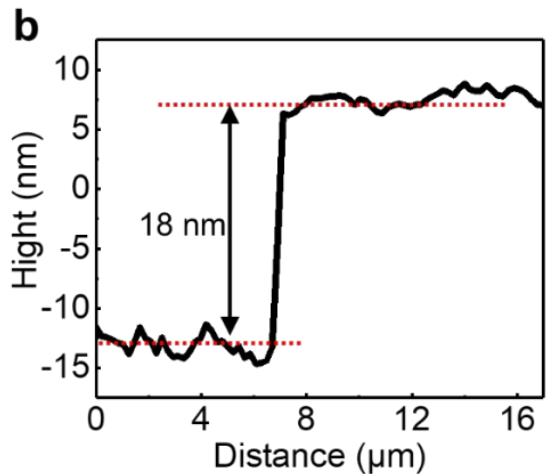

Supplementary Figure 10. a, AFM measurement of a PVA step on top of $\mathrm{SiO}_{2}$ substrate. $\mathbf{b}$, The PVA functionalization layer is $18 \mathrm{~nm}$ thick. 

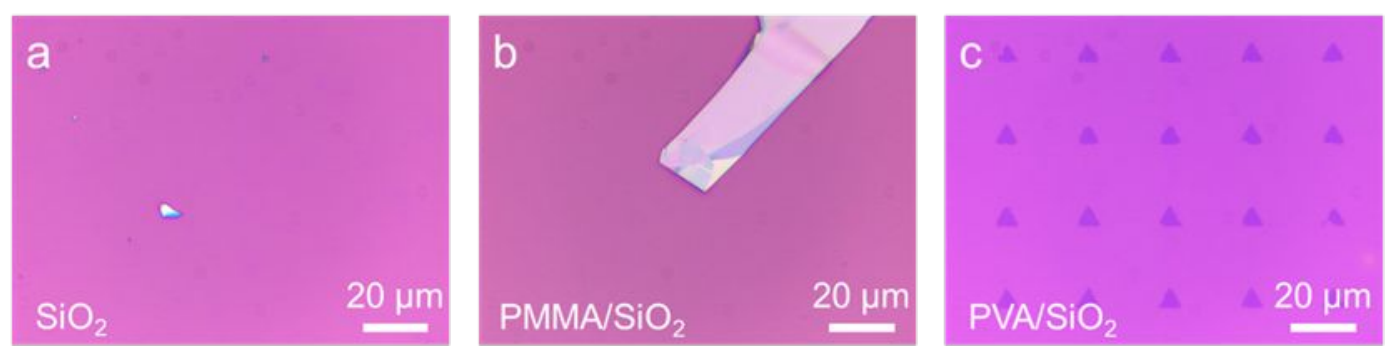

Supplementary Figure 11. Dry exfoliation yield of substrate with or without functionalization layer. a, Optical image of dry exfoliation on bare $\mathrm{SiO}_{2}$ substrate, limited bulk materials are released. b, Optical image of dry exfoliation on PMMA functionalized $\mathrm{SiO}_{2}$ substrate, undesired bulk crystals are released and no monolayer arrays. c, Optical image of dry exfoliation on thin $\mathrm{PVA}$ functionalized $\mathrm{SiO}_{2}$ substrate, high releasing yield is achieved for monolayer arrays. 